\section{Gastrointestinal metastasis of cardiac leiomyosarcoma}

A 78-year-old woman was admitted to our hospital with a 1-month history of fever. Two years earlier, she had undergone surgery for cardiac leiomyosarcoma in the left atrium. Pathological examination had shown complete resection of the tumor.

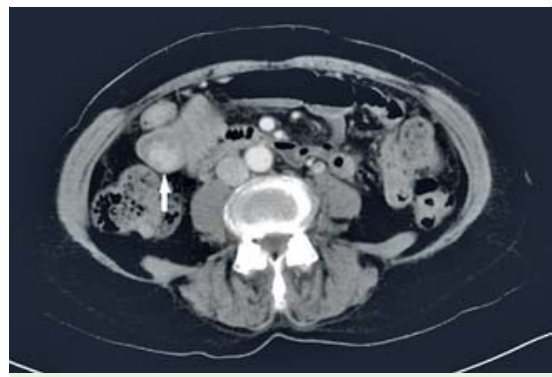

Fig. 1 Contrast-enhanced computed tomography showed an enhanced lesion in the jejunum (arrow).
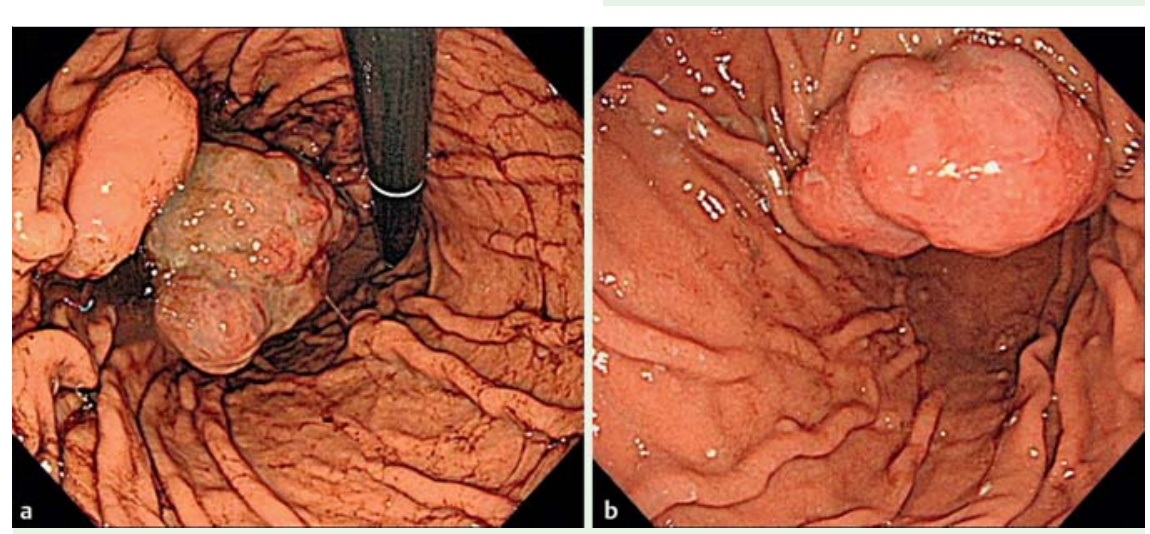

Fig.3 Endosopic view showing the gastric tumors.
Contrast-enhanced computed tomography showed an enhanced structure in the jejunum ( $\bullet$ Fig. 1 ). Transoral singleballoon enteroscopy performed for closer examination detected a smooth-surfaced tumor ( $\bullet$ Fig. 2). Similar tumors were

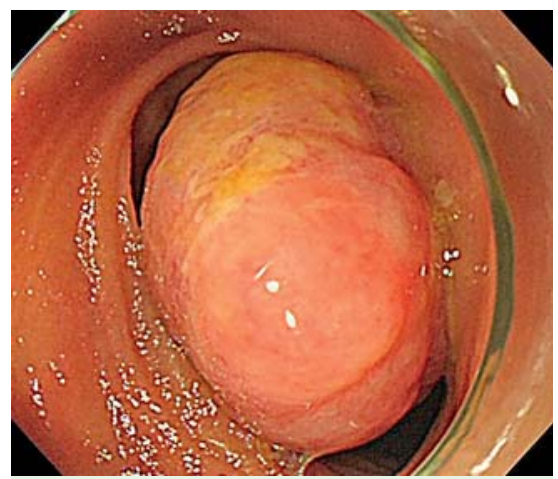

Fig. 2 Endoscopic view showing the jejunal tumor. identified in the stomach ( $\mathbf{F i g . 3}$ ). Elective surgery was scheduled, but intussusception developed, caused by the jejunal tumor, and an emergency operation was performed.

The pathological findings showed spindleshaped cells with high rates of nuclear pleomorphism and mitosis ( $\bullet$ Fig. 4 a). Immunohistochemical studies showed positive results for desmin, $\alpha$-smooth muscle actin, HHF-35, caldesmon, and calponin, but negative results for myoglobin, myogenin, and MyoD1 ( $\bullet$ Fig.4b). The pathological result closely resembled that of the cardiac tumor resected 2 years earlier.

From the above, we concluded that the tumors represented gastrointestinal (GI) metastases of cardiac leiomyosarcoma. The patient declined chemotherapy and was discharged; she remains under follow-up in the outpatient department. Primary cardiac tumors are very rare, with a reported incidence of about $0.02 \%$ per person-year [1]. The frequency of primary leiomyosarcoma of the heart is less than $0.25 \%$ of all cardiac tumors [2]. The prognosis for patients with malignant heart tumors remains poor because treatment approaches have yet to be established. Some reports have described longer survival with complete resection than with incomplete resection [3]. The effectiveness of chemotherapy and/or radiation therapy is controversial [3]. Fewer reports have been made regarding cardiac leiomyosarcoma. Glaoui et al. encountered a long-term survivor, but most patients die within a year [4]. A single-center review revealed no cases of GI metastasis from primary cardiac malignancy [5]. To the best of our knowledge, the case described here represents the first report of GI metastasis of cardiac leiomyosarcoma.

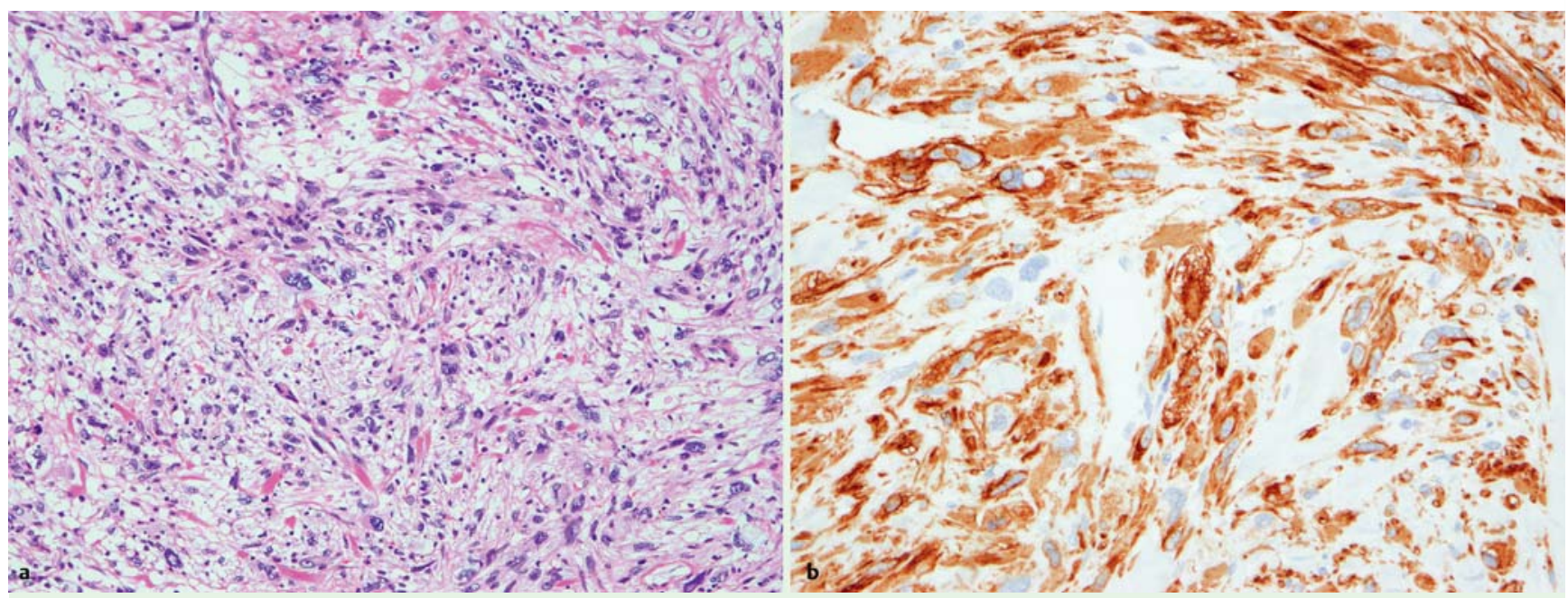

Fig.4 Pathological findings of the jejunal tumor. a Hematoxylin and eosin staining. b Desmin staining. 


\section{Endoscopy_UCTN_Code_CCL_1AC_2AC}

Competing interests: None

Koichiro Abe ${ }^{1}$, Kazunori Seo ${ }^{1}$, Minami Yagi $^{1}$, Yuko Sasajima ${ }^{2}$, Takahiro Yagi ${ }^{3}$, Tsuyoshi Inaba ${ }^{3}$, Ryoji Fukushima ${ }^{3}$

${ }^{1}$ Department of Internal Medicine, Teikyo University School of Medicine, Tokyo, Japan

2 Department of Pathology, Teikyo University School of Medicine, Tokyo, Japan

${ }^{3}$ Department of Surgery, Teikyo University School of Medicine, Tokyo, Japan

\section{References}

1 Reynen $K$. Frequency of primary tumors of the heart. Am J Cardiol 1996; 77: 107

2 Burke AP, Cowan D, Virmani R. Primary sarcomas of the heart. Cancer 1992; 69: $387-$ 395

3 Putnam JB Jr, Sweeney MS, Colon R et al. Primary cardiac sarcomas. Ann Thorac Surg 1991; 51: 906 -910

4 Glaoui M, Benbrahim Z, Belbaraka R et al. An uncommon long-term survival case of primary cardiac leiomyosarcoma. World J Surg Oncol 2014; 12: 338

5 Simpson L, Kumar SK, Okuno SH et al. Malignant primary cardiac tumors: review of a single institution experience. Cancer 2008; 112: $2440-2446$
Bibliography

Dol http://dx.doi.org/ 10.1055/s-0042-118178 Endoscopy 2016; 48: E349-E350

(c) Georg Thieme Verlag KG Stuttgart · New York ISSN 0013-726X

\section{Corresponding author}

\section{Koichiro Abe, MD, PhD}

Department of Internal Medicine Teikyo University School of Medicine 2-11-1 Kaga

Itabashi-ku

Tokyo 173-8606

Japan

Fax: +81-3-53751308

abe@med.teikyo-u.ac.jp 